\title{
Analysis of vortex ring formation in the heart chamber by instantaneous vortex deviation based on convolutional neural network
}

\author{
Ke Yang ${ }^{1}$, Shiqian $\mathrm{Wu}^{2, *}$, and Kelvin K.L. Wong ${ }^{3}$ \\ ${ }^{1}$ School of Machinery and Automation, Wuhan University of Science and Technology, Wuhan, China \\ ${ }^{2}$ School of Information Science and Engineering, Wuhan University of Science and Technology, Wuhan, China \\ ${ }^{3}$ Shenzhen Institutes of Advanced Technology, Chinese Academy of Sciences, Shenzhen, China
}

\begin{abstract}
The formation of vortex rings during the left ventricle (LV) filling is an optimized mechanism for blood transport, and the vorticity is an important measure of a healthy heart and LV. There is a relationship between abnormal diastolic vortex structure and impaired LV, and hence vortex identification is vital for understanding the underlying physical mechanism of blood flow. However, due to lack of quantitative methods, defining, computing and mapping the left ventricular vortices has not been rigorously studied previously. In this paper, a novel method of vortex detection based on the convolutional neural network $(\mathrm{CNN})$ is created, which enables determination of the boundary of vortex and integrates the local and global flow fields. We have used the CNN-based vortex identification and vector flow mapping (VFM) to quantify left ventricular vorticity. In the clinical application of our methodology to healthy subjects and uremic patients, we find differences in the strength and position of the vortices between healthy and patients with uremia cardiomyopathy. Our results can accurately indicate the role of vortex formation in intracardiac flow, and provide new insights into the blood flow within the heart structure.
\end{abstract}

\section{INTRODUCTION}

Left ventricle (LV) fluid dynamics presents a complex flow phenomenon. During the LV filling, the blood flow begins with the transmitral flow achieving a maximal velocity of about $100 \mathrm{~cm} / \mathrm{s}$ into the $\mathrm{LV}$. In less than a second, the blood flow in LV changes direction and leaves the LV into the ascending aorta with a maximal velocity of about $66 \mathrm{~cm} / \mathrm{s}$. During the course of an optimized flow path, a vortex flow is developed during diastole, to redirect the blood flow toward the LV outflow tract. ${ }^{[1]}$ Earlier studies have shown that the vorticity is an important measure of LV diastolic function and overall heart health. ${ }^{[1]}$ Therefore, quantitative measurements of vorticity can provide deeper insights into hemodynamics within the heart.

Earlier studies have quantified the vorticity based on the different time frames of a cardiac cycle. ${ }^{[1,2]}$ Wong et al have measured vortex characteristics by calculating the vorticity and developing two-dimensional vortical flow maps. ${ }^{[2]}$ Kheradvar et al analyzed velocity sequence obtained by ultrasonic electrocardiogram, to derive further LV spatial flow characteristics for better characterization of the flow patterns, and used the vorticity to describe blood motion. ${ }^{[1]}$ Most of these methods are based on a finite-differences approach, and the evaluation of vorticity has been performed globally in the region of interest after segmentation, which has suffered from loss of accuracy in the irregular LV boundary and ignoring of the main component of the vortex. Hence accurate identification of vortices is important for studying the law and mechanism of blood flow in the LV.

Vortex identification methods based on associated implicit vortex definition have been developed, and these methods can be roughly classified into two categories:

local methods and global methods. ${ }^{[3]}$ The local methods are generally based on physical properties of the flow field, and only use the local flow information to compute some criterions and obtain the results efficiently. However, there are many false positives and false negatives in the results. Different from the local methods, the global methods are typically based on global topological properties of the flow field, such as Lagrangian-Averaged Vorticity Deviation (LAVD). These methods use global flow information to detect vortical regions, and have excellent objectiveness and robustness. ${ }^{[4]}$ However, global methods are computationally intensive, and therefore take more time than local methods.

Since the local and global methods have their advantages and disadvantages, it is worth combing the advantages of both the methods. Meanwhile, defining the boundary of the vortex in the LV poses a challenge, because the flow is intricate and involves a large-scale data set. The complex blood flow makes local methods have a high error rate, and the global methods are

\footnotetext{
* Corresponding author: shiqian.wu@wust.edu.cn
} 
difficult to deal with the large size of data. With this in mind, we have innovatively adopted the vortex identification method based on convolution neural network $(\mathrm{CNN})$ taking into account the local and global information of the LV flow field to solve these problems. Additionally, we have sought to quantify the vorticity after vortex identification, and to compare differences between healthy volunteers and patients with uremia cardiomyopathy. These sample cases have demonstrated the working principles of our proposed framework, that is devised to perform component flow analysis and justify the effectiveness of the methodology.

\section{Methods}

\subsection{Study population}

Ten healthy volunteers and five patients with uremic cardiomyopathy were included in our study. All healthy volunteers had normal blood pressure, normal ECG, and no history of cardiovascular disease. The study was approved by the Regional Ethical Review Board of the Third Affiliated Hospital of Sun Yat-sen University. All subjects underwent cardiovascular ultrasound, consisting of cine images in 2-chamber, 3-chamber, 4-chamber, and short-axis views.

\subsection{Echocardiography}

The ultrasound device is Prosound F75 ultrasound (Hitachi Aloka medical Ltd., Tokyo, Japan). The apical long-axis view was adopted for acquiring the color Doppler images, by using a UST-52105 probe through a water bag, at a transducer frequency of $5 \mathrm{MHz}$, depth of $8 \mathrm{~cm}$, focal point of $5 \mathrm{~cm}$, and frame rate of 29-57. The images were captured over three successive heartbeats and stored on a built-in hard disk. Then, the Vector Flow Mapping (VFM) analysis software (DAS-RS1, Hitachi Aloka Medical Ltd) was employed for vector flow mapping, by flow visualization echocardiographic technology. The relationship between ECG and cardiac cycle defines the cardiac events based on the number of scan time frames.

\subsection{Convolution neural network for vortex identification}

The CNN-based vortex identification method consists of two parts: the data processing part and the network part, ${ }^{[5]}$ as shown in Fig. 1. The data processing part provides the data inputs to the second part, and the network part trains a CNN model to recognize the vortical structures and define the boundary of the vortexes in the flow field of the LV.

\subsubsection{The data processing part}

The data processing part of the CNN-based vortex identification includes four steps: acquiring LV velocity data, obtaining labels, normalization, and sampling patches.

Firstly, the LV velocity data can be expressed as rectangular arrays, and each point has associated velocity components and location information in the Cartesian coordinate system.

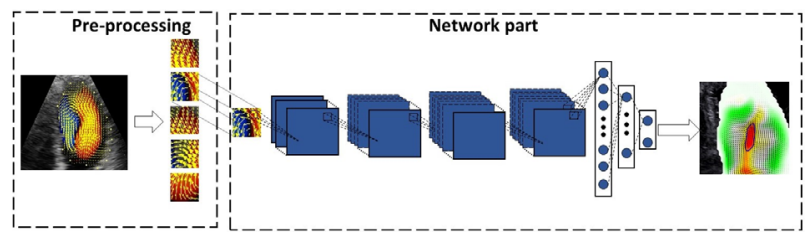

Fig. 1. The architecture of CNN-based LV vortex identification.

Secondly, CNN is a typical supervised learning algorithm, which requires a large amount of labelled data to train the network. A global vortex method named instantaneous vorticity deviation (IVD) can be used to label all points in the LV flow field. ${ }^{[4]}$ Haller et al. have defined vortices as sets of tubular surfaces of constant intrinsic material rotation rate and proposed LAVD, a threshold-dependent vortex identification technique. ${ }^{[4]}$ The IVD, the transient expression of LAVD, is described as the absolute value of the difference between the vorticity at a point and the space average vorticity of the global field:

$$
\operatorname{IVD}(x, t)=\left|\omega(x, t)-\omega_{\operatorname{avg}}(x, t)\right|,
$$

Where $w$ represents the vorticity value, and $x$ is the position of the flow field. The IVD can obtain the definition of the instantaneous vortex and describe the boundary of vortex as the largest convex member of nested tube family. The IVD also has the ability to detect vortical features more quickly and accurately in $2 \mathrm{D}$ and 3D flows. ${ }^{[4]}$ The Training data is based on the LV blood flow of patients, and can be labelled by the IVD. Note that ' 0 ' represents the point outside the boundary of the vortex, and ' 1 ' represents the point inside the boundary of the vortex. In this way, the LV blood flow global information can be obtained.

Thirdly, the velocity rectangular arrays need to be normalized, and the normalization method is shown in Eq. (3):

$$
u=\frac{\left(u_{0}-u_{\min }\right)}{\left(u_{\max }-u_{\min }\right)} ; \quad v=\frac{\left(v_{0}-v_{\min }\right)}{\left(v_{\max }-v_{\min }\right)},
$$

where $u$ and $v$ are the normalized velocity components of each points in the LV flow field; $u_{0}$ and $v_{0}$ are the original velocity; $u_{\max }, u_{\min }, v_{\max }, v_{\min }$ are the maximum and minimum of the velocity components of each points in the original LV flow field, respectively.

Finally, the normalized velocity field needs to be sampled by local patches around each of the points, and these patches and labels are used as input of the network, as shown in Fig. 1. By using local patches and global labels to train the network, the CNN method combines global and local LV flow information in the testing stage. 


\subsubsection{Structure of the network}

After the data processing part, each point must be classified. The classification task is performed by the network part, which trans a CNN model to distinguish non-vortex points and vortex points, and define the boundary of the vortex in the LV. The network includes a single input layer, four convolutional layers, and three fully connected layers, as shown in Fig. 1. The first layer is the input layer corresponding to a patch in the LV flow field. The size of this input layer is $2 \times 19 \times 19$, in which 2 represents the number of channels and 19 is the size of patches. The second to the fifth layer are convolutional layers all of which use the convolution kernels by $3 \times 3$. These kernels need to be decided through the training stage and the number of feature maps of these layers is 8, 16, 32 and 64. Meanwhile, these convolutional layers use the activation function is the rectified linear unit (ReLU), as shown in Eq. (3):

$$
y=\max (0, x),
$$

wherein $y$ means the output of the activation function, and $x$ represents the output of the convolutional layer. The sixth to eight layers are the fully connected layers with 128, 64 and 2 neurons, respectively. The last fully connected layer is the output layer, which describes the boundary of the vortex and classifies the input into 'vortex' or 'non-vortex'. The output layer is a SoftMax layer, where each neuron means a probability of the input belonging to one category. The SoftMax neuron is given by:

$$
\begin{gathered}
y_{i}=\frac{e^{z_{i}}}{\sum_{j=1}^{m} e^{z_{j}}} \\
z_{j}=\sum_{k=1}^{N_{\text {prev }}}\left(w_{k, j} x_{k}\right)+b,
\end{gathered}
$$

where $m$ represents the number of classes, $z_{j}$ is the output of the last layer, $N_{P R V E}$ represents the number of neurons of previous layer, $W_{k, j}$ and $x_{k}$ are weight and output the kth neuron of the previous layer to the jth neuron of the SoftMax layer. ${ }^{[5]}$

The Network uses back-propagation with the crossentropy cost function to optimize the parameters. The cross-entropy cost function is shown in Eq. (6):

$$
c=-\frac{1}{n} \sum_{x}[y \operatorname{In} a+(1-y) \operatorname{In}(1-a)]
$$

where (i) $x$ and $n$ are the number of training samples and the total number of training samples, and (ii) $y$ and $a$ represent the predicted and true output of the neuron. Back-propagation iteratively calculates the gradient of each layer by using chain rule and starting from the output layer. The well-trained CNN model can thus be obtained and employed to correctly identify the vortex.

\section{Results}

In this section, we implement the method based on the CNN to identify the vortex in the LV during the complete cardiac filling process, compute and map the spiraling flow fields, and compare them for healthy people and patients with uremia cardiomyopathy. We use an in-house data set that contains $400 \mathrm{LV}$ velocity rectangular arrays attached with associated labels in different states to train the $\mathrm{CNN}$ model. The trained CNN model is employed to identify the LV vortex of ten healthy volunteers and five patients with uremia cardiomyopathy. Fig. 2 illustrates the computed LV flow visualizations of one healthy person and one uremic patient, during (i) rapid filling, (ii) the end of rapid filling, (iii) reduced filling, and (iv) atrial systole.

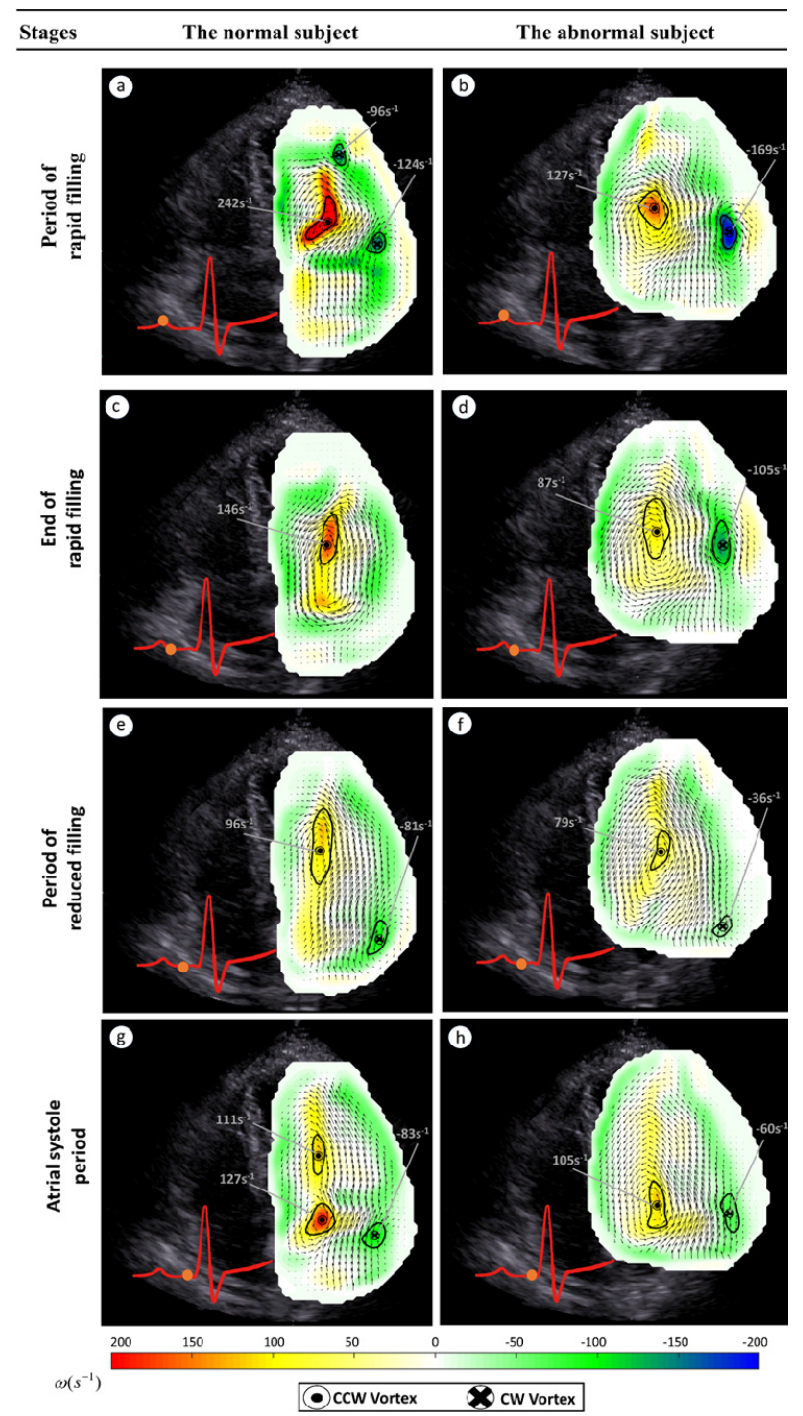

Fig. 2. Flow visualizations of normal and abnormal LV, during (i) rapid filling, (ii) the end of rapid filling, (iii) reduced filling, and (iv) atrial systole.

In Fig. 3, we are depicting the strengths of two vortices in opposite directions of ten healthy volunteers and five patients during the LV diastolic period. This provides evidence that the physical flows are related to the cardiac events, and the uremia cardiomyopathy disease leads to differences in vorticity between patients 
and healthy people. Although there are the outliers, which are caused by mismatching the flow patterns or the excessive resolution during the acquisition process. It can be observed that the vorticity of healthy volunteers is generally higher and positive (dominant vortex is counter-clockwise rotation) during the LV diastole. At the transition stage to LA systole, the vorticity values keep decreasing. In the LA systolic event, the dominant vortex appears at the basal level of the LV, and the vorticity is increased. The flow patterns and properties of patients with uremia cardiomyopathy are more complex than of the healthy group.

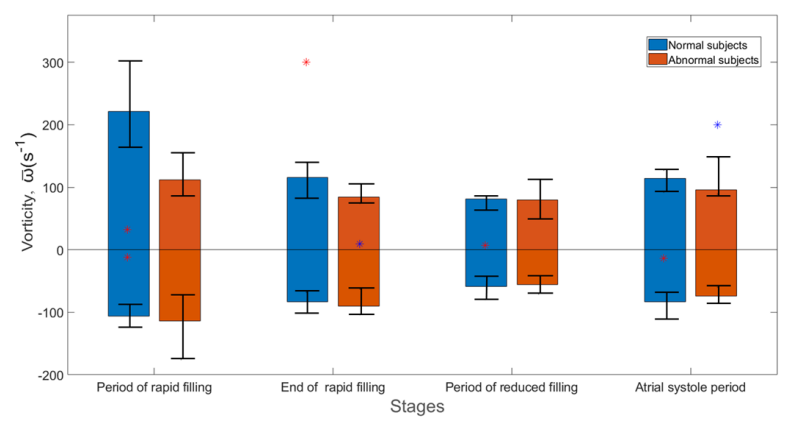

Fig. 3. Comparison of vorticity between healthy group and patients with uremia cardiomyopathy at each stage of LV filling. ' ${ }^{*}$ ' represents the outliers.

In this paper, we use two classical metrics to measure the performance of $\mathrm{CNN}$-based vortex identification method at various periods of LV filling, including recall and precision. They are calculated by using Eq. (7):

$$
\text { recall }=\frac{T P}{T P+F N} ; \quad \text { precision }=\frac{T P}{T P+F P},
$$

where TP, FP, TN and FN represent the number of true positives, false positives, true negatives and false negatives, respectively.

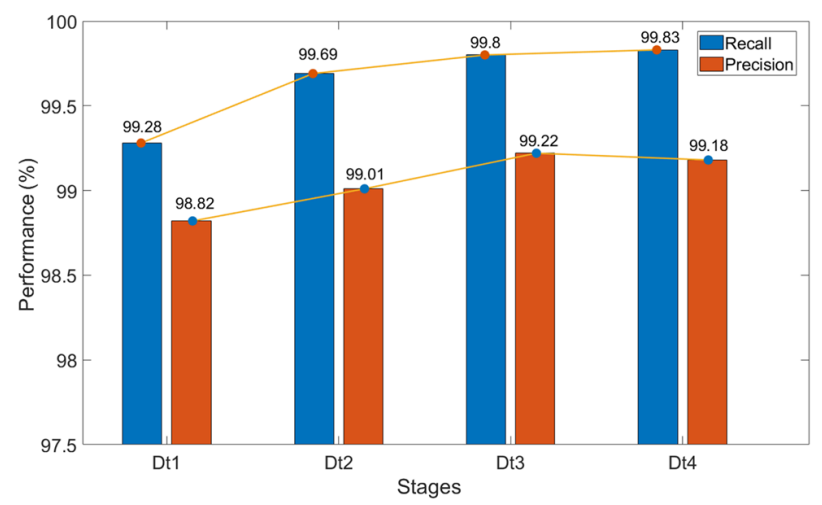

Fig. 4. The performance of CNN-based vortex identification at each stage of LV.

The curves of recall and precision over time are shown in Fig. 4. In terms of the temporal variation of recall and precision, the trend is from growth to stability, which may be due to the relatively complex blood flow at the beginning of filling. However, recall and precision are always more than $98.5 \%$, which means that (i) there are lots of true positives and a small number of false negatives and false positives in the vortex identification results, and (ii) the $\mathrm{CNN}$ model can achieve a high recall as well as high precision during the LV filling period.

\section{Discussion}

The objective of this study is to identify blood flow vortices in the LV, and measure the difference of vorticity between healthy people and patients during the LV diastole, by combing the local and global blood flow field information and adopting CNN. From an innovative perspective, the LV vortex identification issue is transformed into binary classification. Breaking down the vorticity analysis into examination of individual vortices allows us to characterize the blood flow behavior more accurately.

We find that at the beginning of ventricular diastole, the identified vortices are both counter-clockwise and clockwise in ten healthy volunteer and five patients. This is in line with an earlier study by Johannes et al. ${ }^{[6]}$ while (i) the counter-clockwise vorticity mean of the healthy group is higher than that of patients, and (ii) the clockwise vorticity mean of patients is higher than that of the healthy group. This relatively complicated situation may be a consequence of the complex variation and the asymmetry of flow in the LV. At the middle and end of ventricular diastole, the flow patterns of healthy volunteers are visually same as that of patients, and the defined vortices are counter-clockwise and clockwise (with the dominant vortex being counter-clockwise rotation) in both healthy groups and patients. However, the main difference between the two groups is that vorticity of the healthy subjects is still higher than that of patients, as shown in Fig. 3.

Meanwhile, we can note that our method of identifying the LV vortex based on $\mathrm{CNN}$ has high precision, as shown in Fig.4. Also, the network is threshold-independent, and can provide vortex identification result objectively and robustly, which is a consequence of using IVD and global flow information to label data. Compared with conventional network architecture, our methodology removes the pooling layer, because (i) we have sampled the velocity rectangular arrays in the data processing, and (ii) the size of input data is relatively small, which shows local information of LV flow fields. Based on our results, the CNN model can be seen to accurately detect the vortical structures, and reveal the nature of flow phenomena in the LV flow fields. More importantly, the model trained by one LV case can be directly applied to other cases, thereby avoiding a long time of training, with good universality and scalability.

However, there are some significant works needing to be improved. Firstly, in order to enhance the performance of $\mathrm{CNN}$ model, deeper $\mathrm{CNN}$ architecture can be used. Adopting a highly and complex modular approach to design the CNN model is a clear way to integrate new improved algorithms. Secondly, tracking development of LV vortex is a research area closely related to vortex identification. Future work will focus on labelling the time-dependent flow field and describing the evolution of the vortex feature. 


\section{Conclusion}

In this paper, we have adopted a CNN-based method to identify the LV flow field vortex and map the flow patterns, and have measured the differences in vorticity between healthy and patients with uremia cardiomyopathy to justify effectiveness of the method. The novelty of this study is that we can innovatively detect and measure the LV dominant vortex during the complete LV diastole. The boundary of LV vortex can be defined by using local and global information of flow field, and a CNN model is trained which combines the advantage of traditional vortex identification for the characteristics of LV blood flow. Finally, the vorticity of healthy subjects and patients is calculated based on the result of vortex identification. The quantization of individual vortices can provide a good reference for the early diagnosis of uremia cardiomyopathy.

\section{Acknowledgements}

This work was funded by National Natural Science Foundation of China (Grant Number 81771927).

\section{References}

1. Kheradvar A, Houle $H$, Pedrizzetti $G$, et al. Echocardiographic Particle Image Velocimetry: A Novel Technique for Quantification of Left Ventricular Blood Vorticity Pattern[J]. Journal of the American Society of Echocardiography, 23(1):86-94 (2010).

2. Wong K K L, Kelso R M, Worthley S G, et al. Cardiac Flow Analysis Applied to Phase Contrast Magnetic Resonance Imaging of the Heart[J]. Annals of Biomedical Engineering, 37(8):14951515 (2009).

3. Wu J Z, Xiong A K, Yang Y T. Axial stretching and vortex definition[J]. Physics of Fluids, 17(3):038108 (2005).

4. Haller G, Hadjighasem A, Farazmand M, et al. Defining Coherent Vortices Objectively from the Vorticity[J]. Journal of Fluid Mechanics, 795(7):136-173 (2015) .

5. Deng L, Wang Y, Liu Y, et al. A CNN-based vortex identification method $[\mathrm{J}]$. Journal of Visualization, 22:65-78 (2019).

6. Johannes Töger, Kanski M, Carlsson M, et al. Vortex Ring Formation in the Left Ventricle of the Heart: Analysis by 4D Flow MRI and Lagrangian Coherent Structures[J]. Annals of Biomedical Engineering, 40(12): 2652-2662 (2012). 